\title{
Postorgasmic illness syndrome
}

INSERM

\section{Source}

INSERM. (1999). Orphanet: an online rare disease and orphan drug data base.

Postorgasmic illness syndrome. ORPHA:279947

Postorg asmic illness syndrome is a rare urogenital disease characterized by the appearance of flu-like symptoms (fever, extreme fatigue, myalgia, itchy burning eyes, nasal congestion/rhinorrhea), as well as mood changes, irritability and concentration, memory and attention difficulties, within a few minutes to a few hours after ejaculation. Symptoms disappear spontaneously 3-7 days after onset. 